Review

\title{
State of the Art on Approved Cystic Fibrosis Transmembrane Conductance Regulator (CFTR) Modulators and Triple-Combination Therapy
}

\author{
Aniello Meoli ${ }^{1}$, Valentina Fainardi ${ }^{1}$, Michela Deolmi ${ }^{1}$, Giulia Chiopris ${ }^{1}$, Francesca Marinelli ${ }^{1}$, \\ Caterina Caminiti ${ }^{2} \mathbb{D}$, Susanna Esposito ${ }^{1, *}$ and Giovanna Pisi ${ }^{1}$ \\ 1 Paediatric Clinic, Department of Medicine and Surgery, University Hospital of Parma, Via Gramsci 14, \\ 43126 Parma, Italy; nello.meoli@gmail.com (A.M.); valentina.fainardi@unipr.it (V.F.); \\ michela.deolmi@studenti.unipr.it (M.D.); giulia.chiopris@gmail.com (G.C.); \\ francesca.marinelli@studenti.unipr.it (F.M.); gpisi@ao.pr.it (G.P.) \\ 2 Research and Innovation Unit, University Hospital of Parma, Via Gramsci 14, 43126 Parma, Italy; \\ ccaminiti@ao.pr.it \\ * Correspondence: susannamariaroberta.esposito@unipr.it
}

\section{check for}

updates

Citation: Meoli, A.; Fainardi, V.; Deolmi, M.; Chiopris, G.; Marinelli, F.; Caminiti, C.; Esposito, S.; Pisi, G. State of the Art on Approved Cystic Fibrosis Transmembrane

Conductance Regulator (CFTR) Modulators and Triple-Combination Therapy. Pharmaceuticals 2021, 14, 928. https://doi.org/10.3390/ ph14090928

Academic Editor: Daniela Catarzi

Received: 14 July 2021

Accepted: 10 September 2021

Published: 15 September 2021

Publisher's Note: MDPI stays neutral with regard to jurisdictional claims in published maps and institutional affiliations.

Copyright: (c) 2021 by the authors. Licensee MDPI, Basel, Switzerland. This article is an open access article distributed under the terms and conditions of the Creative Commons Attribution (CC BY) license (https:// creativecommons.org/licenses/by/ $4.0 /)$.
Abstract: Cystic fibrosis (CF) is the most common life-limiting inherited disease in Caucasian populations, affecting approximately 80,000 people worldwide. $\mathrm{CF}$ is a complex multi-organ monogenic autosomal recessive disorder caused by a mutation in cystic fibrosis transmembrane conductance regulator (CFTR) gene. Since the discovery of the CFTR gene in 1989, more than 2000 mutations have been identified so far and about 240 can cause CF. Until recently, the treatment for CF was aimed to prevent and manage the manifestations of CFTR dysfunction, primarily recurrent pulmonary infections and pancreatic exocrine failure. Over the past few decades, the therapeutic approach to CF has been revolutionized by the development of a new class of small molecules called CFTR modulators that target specific defects caused by mutations in the CFTR gene. CFTR modulators have been shown to change profoundly the clinical course of the $\mathrm{CF}$, leading to meaningful improvements in the lives of a large proportion of people of CF heterozygous for F508del, especially if started in young children. Further studies are needed to extend the use of triple CFTR modulation therapy also for young children in order to prevent the irreversible effects of the disease and for patients with very rare mutations with a personalized approach to treatment.

Keywords: CFTR; CFTR modulator; cystic fibrosis; F508del; ivacaftor

\section{Introduction}

Cystic fibrosis (CF) is the most common life-limiting inherited disease in Caucasian populations, affecting approximately 80,000 people worldwide $[1,2]$. CF is a complex multiorgan monogenic autosomal recessive disorder caused by a mutation in cystic fibrosis transmembrane conductance regulator (CFTR) gene on the long arm of chromosome 7 [2]. This gene encodes for an anion channel member of the ATP-binding cassette $(A B C)$ proteins regulated by cyclic adenosine monophosphate (cAMP) and protein kinase A (PKA)-dependent phosphorylation responsible for chloride and bicarbonate passive transport through the apical membrane of epithelial cells. The CFTR protein is a is 1480 amino acid glycoprotein located on secretory epithelia of airways, pancreas, sweat glands, and the gastrointestinal and reproductive tracts, also involved in the modulation of other adjacent ion channels and therefore crucial for the homeostasis of these organs due to its ability to regulate epithelial surface hydration and luminal $\mathrm{pH}$. A defective CFTR function causes a reduced anion secretion through the lumen of airways and digestive system with consequent impaired hydration of their secretions. In addition, in airways, sodium absorption, of which the CFTR protein is a down-regulator, contributes to the surface dehydration and impaired mucociliary clearance observed in CF patients. By contrast, CFTR mutation in sweat glands 
causes a reduction in chloride and sodium absorption through the cytoplasm with higher sodium chloride levels in sweat. Hence, impaired function of the CFTR gene causes a wide range of symptoms, including thickened mucous with formation of mucus plugs, severe lung infections, pancreatic insufficiency, liver damage, increased sweat chloride concentration, malabsorption with malnutrition, and infertility [2]. Airways involvement with recurrent pulmonary exacerbations, especially by Pseudomonas aeruginosa infection that led to progressive lung damage, represents the most frequent cause of early morbidity, death, and need for lung transplantation in CF patients [2]. The reduced bicarbonate secretion in pancreatic ducts of CF patients leads to an increased mucus viscosity and decreased solubility of secreted digestive enzymes with premature activation of them and consequent pancreas destruction [2]. The loss of pancreatic exocrine function leads to the need to take enzyme supplements; over time, the pancreatic damage also affects the endocrine function of these patients that may develop insulin-dependent diabetes mellitus. In addition, the reduced secretion of bicarbonate is also present in the intestinal lumen with increased acidity and consequent epithelial damage and dehydration, which can lead to intestinal obstruction [2]. In neonates, intestinal obstruction may cause meconium ileus, while in adults, it may result in recurrent episodes of distal intestinal obstructive syndrome (DIOS) [2]. Therefore, intestinal dysbiosis may cause chronic diarrhea and weight loss. Figure 1 summarizes the clinical manifestations in patients with CF.

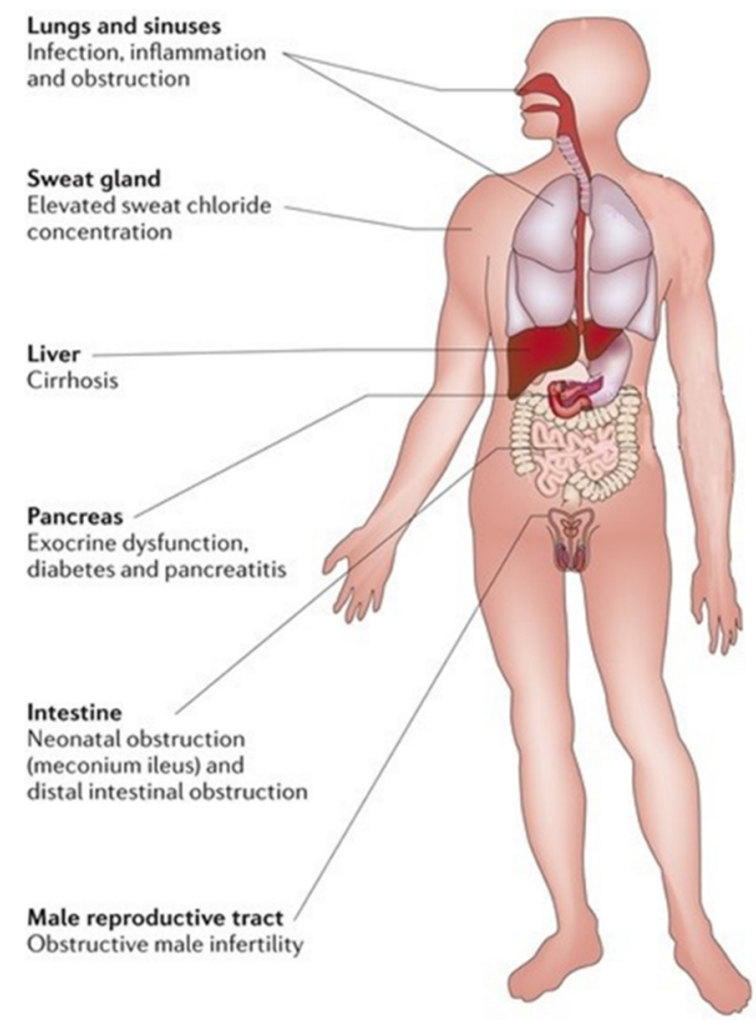

Figure 1. Clinical manifestations of cystic fibrosis.

Since the discovery of the CFTR gene in 1989, more than 2000 mutations have been identified so far and about 240 can cause CF [3]. Based on the pathophysiological effects of individual variants, they have been classified into seven classes (Figure 2): class I, severely reduced protein synthesis with absent CFTR gene expression on the apical membrane; class II, impaired protein processing, folding, or trafficking, and premature degradation with severely reduced CFTR gene expression, such as F508del, the most common mutation, especially in North European countries (80-90\%); class III, CFTR gene can reach the apical membrane, but the channel activation is abnormal (gating mutations); class IV, normal CFTR gene expression with reduced conductivity; class V, lower CFTR gene 
expression due to reduced protein synthesis; class VI, lower CFTR gene expression due to increased turnover; class VII, no mRNA synthesis and consequently no CFTR gene expression [3-5]. There is a correlation between CFTR gene function and clinical phenotype, with classes I-III and VII resulting in more severe disease, while classes IV-VI may have milder phenotype [6].

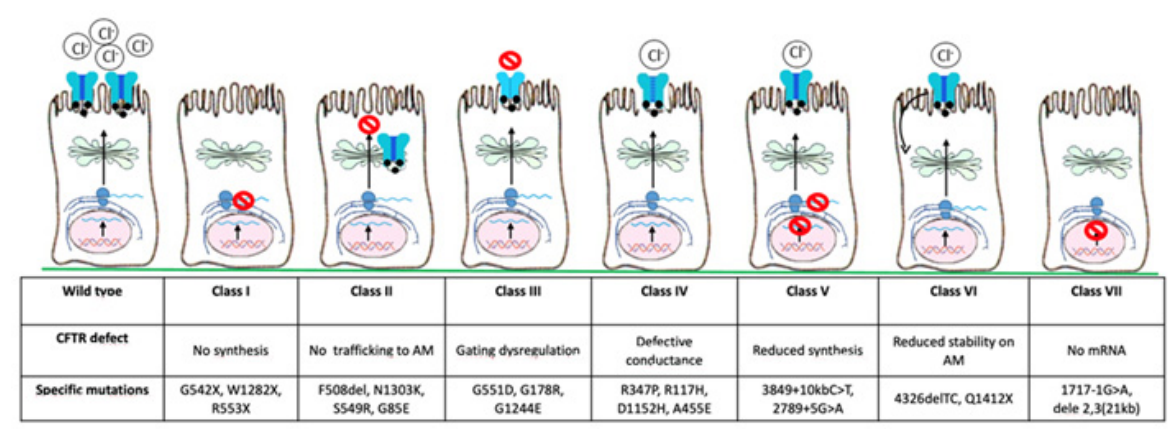

Figure 2. CFTR gene mutations are classified into seven main classes based on the defect of CFTR gene. AM, apical membrane.

Until recently, the treatment for CF was aimed to prevent and manage the manifestations of CFTR gene dysfunction, primarily recurrent pulmonary infections and pancreatic exocrine failure [4]. The major and life-threatening consequences of CF are pulmonary infections and progressive lung function decline. Mucociliary clearance is improved by airway clearance techniques and inhaled mucolytics such as dornase alpha (rhDNase) and hypertonic saline, while chronic pulmonary infections are treated with inhaled, oral, and parenteral antibiotics [4]. With the onset of respiratory failure, lung transplant may represent the last therapeutic option. Pancreatic insufficiency is managed by pancreatic enzyme replacement therapy (PERT) and a highly caloric and salty diet, with the supplementation of fat-soluble vitamins; insulin may be needed in patients who develop diabetes mellitus or glucose intolerance [1]. With improved management, life expectancy for people with $\mathrm{CF}$ has progressively increased and the median predicted life expectancy is now nearly 50 years [7].

Over the past few decades, the therapeutic approach to $\mathrm{CF}$ has been revolutionized by the development of a new class of small molecules called "CFTR modulators" that target specific defects caused by mutations in the CFTR gene. According to their mechanism of action, CFTR modulators can be classified as potentiators (i.e., drugs improving the ions flux through CFTR channel already expressed on apical membrane maintaining it in an open status, effective in patients with gating class III and IV mutations), correctors (i.e., drugs facilitating CFTR protein folding, processing, and trafficking to the cell surface, effective in patients with class II mutations), amplifiers (i.e., drugs increasing the expression of protein substrate [CFTR mRNA] and consequently, synthesis of CFTR protein), readthrough agents (i.e., drugs promoting the ribosomal over-reading of premature termination codons [PTCs, Class I mutations] enabling the incorporation of an amino acid, thereby increasing the production of CFTR proteins), and stabilizers (i.e., drugs rescuing the protein stability at the plasma membrane) $[8,9]$.

This paper aims to critically analyze the role of CFTR modulators in CF treatment. We review PubMed literature published from April 2016 to May 2021, using the keywords "cystic fibrosis", "CFTR modulators", and "combination therapy". We screened 122 articles and abstracts and then we focused on reviews, meta-analysis, and original articles including randomized controlled trials (RCT) and preclinical studies of potentiators and correctors. 


\section{CTFR Potentiators}

\subsection{Ivacaftor (Formerly VX-770; KALYDECO ${ }^{\circledR}$ )}

Ivacaftor (formerly VX-770, Figure 3) was the first CFTR modulator approved by the Food and Drug Administration (FDA) in 2012 with the name of Kalydeco ${ }^{\circledR}$ for the treatment of CF patients heterozygous for the G551D, the most common variant of class III. The mechanism of action of Ivacaftor consists of increasing the time of CFTR channel in open status through the CFTR channel potentiation in a phosphorylation-dependent and ATP-independent manner [10]. Ivacaftor has now been approved for patients older than 4 months with certain class III (G551D, G1244E, G1349D, G178R, G551S, S1251N, S1255P, S549N, S549R) or class IV mutations (R117H), which account for $8 \%$ of all patients with CF $[11,12]$.<smiles>CC(C)(C)c1cc(C(C)(C)C)c(NC(=O)c2c[nH]c3ccccc3c2=O)cc1O</smiles>

Figure 3. Chemical structure of Ivacaftor.

The first double-blind randomized controlled trial (RCT; STRIVE, NCT00909532) was conducted in 2011 by Ramsey et al. to assess the efficacy of Ivacaftor in 161 patients older than 12 years with at least one G551D mutation for 48 weeks. At week 24, the authors found an improvement of percent predicted Forced Expiratory Volume at 1st second (ppFEV1) by $10.6 \%$ in the Ivacaftor group. In addition, they also demonstrated a significant reduction in pulmonary exacerbations $(-55 \%)$, an improvement in patient-reported respiratory symptoms measured by the Cystic Fibrosis Questionnaire-Revised (CFQ-R), a weight gain on average of $2.7 \mathrm{~kg}$ per patient, and a reduction in the concentration of sweat chloride ( $-48.1 \mathrm{mmol}$ per liter). The incidence of adverse events (AE) was similar with Ivacaftor and placebo, with a lower proportion of serious adverse events (SAE) in Ivacaftor group than placebo group (24\% vs. $42 \%$ ) [13]. Two years later, Ivacaftor was tested among $52 \mathrm{CF}$ children aged 6-11 years with at least one G551D mutation (ENVISION trial, NCT00909727), showing the same promising results observed in adult patients: significant increase in ppFEV1 $(+12.6 \%$ in the treatment group), weight gain, and reduction in sweat chloride concentration. Compared to placebo, patients treated with Ivacaftor showed no higher risk of AEs. Unlike the STRIVE study, the ENVISION trial did not show a significant improvement in either CFQ-R score or rate of pulmonary exacerbations $[11,14]$.

Thereafter, De Boeck et al. explored the efficacy of Ivacaftor in patients older than 6 years with nine different non-G551D gating mutation in the KONNECTION study (NCT1614470) [15]. After eight weeks of Ivacaftor, authors reported a significant increase in ppFEV1 in the Ivacaftor group compared to the placebo group (+10.7\%). A significant improvement was also observed in body mass index (BMI) as well as in concentrations of sweat chloride and CFQ-R scores. These results were maintained through 24 weeks and Ivacaftor was generally well tolerated. This trial suggested that CF patients with non-G551D gating mutations could also benefit of treatment with Ivacaftor [15].

The KONDUCT trial (NCT01614457) assessed the safety and efficacy of Ivacaftor in 69 CF patients aged 6 years or older with at least one copy of the R117H-CFTR mutation, which is present in 3\% of all CF patients and produces defects in both gating and conductance (class III and IV mutations, respectively) [16]. After 24 weeks of treatment, significant improvements from baseline were found of either CFQ-R score and sweat chloride levels in Ivacaftor group. In contrast, no significant difference from baseline was seen in either ppFEV1 or BMI. However, subgroup analysis based on age showed 
significant improvement in ppFEV1 in the Ivacaftor group only for patients older than 18 years. These contrasting results between adults and children may be due to the intrinsic nature of R117H-CFTR mutation, characterized by a lower degree of severity in children and a delayed onset of significant disease involvement, typically in adulthood [16].

The KIWI trial (NCT01705145), a phase 3 open label study, explored efficacy and safety of Ivacaftor for 24 weeks in 33 children aged 2-5 years with a gating mutation [17]. A significant improvement in sweat chloride concentration and nutritional status was observed, similar to that seen in adult population. No statistical relevant data on pulmonary effect were available due to small age of patients. At baseline, fecal elastase-1 was insufficient in $96.3 \%$ of patients but increased above the cut-off for pancreatic insufficiency in $25 \%$ of them, suggesting that when started early in life, Ivacaftor could potentially restore exocrine pancreatic function. A higher incidence of liver function test (LFT) elevation was reported in this pediatric study than in adult ones [17].

The KLIMB study (NCT01946412) included 28 of the 33 children enrolled in the KIWI trial and followed them up for a total of 84 weeks [18]. This long-term extension trial proved that Ivacaftor was generally well tolerated in CF children 2 to 5 years old, although even in this study, $30 \%$ of children experienced an LFT elevation $>3 \times$ Upper Limit of Normal (ULN) at least on one occasion. Only one child experienced value of alanine aminotransferase $>8 \times$ ULN and required treatment discontinuation. Consistently with the KIWI study, LFT elevation occurred more often in patients with a previous history of transaminase elevation; therefore, authors recommended LFT monitoring before and during treatment with Ivacaftor in children aged 2-5 years. During the 84 weeks of observation, improvements in sweat chloride concentrations, BMI z-scores, and pancreatic function as recorded in the KIWI trial were generally maintained [18].

Safety and efficacy of Ivacaftor in children aged 12-24 months (26 children with at least one CTFR gating mutation) was assessed by the ARRIVAL study (NCT02725567), published in 2019 [19]. Oral Ivacaftor was administrated for 24 weeks with rapid and sustained improvement in sweat chloride concentration. Growth parameters were normal at baseline and at week 24. Furthermore, authors observed a significant improvement in fecal elastase1 and a decrease from baseline in serum lipase and amylase. Once again, improvements in biomarkers of pancreatic function suggest that Ivacaftor, if started early, may preserve exocrine pancreatic function. The most frequently AEs were cough and transaminase elevation; one child suffered from constipation, possibly related to the modulator. No treatment discontinuation or interruption occurred.

Based on in vitro studies, another extension of Ivacaftor has been recently approved by FDA to include CFTR gene mutations with residual function (RF), totalizing 28 eligible CFcausing mutations and increasing the number of patients who may benefit from Ivacaftor treatment [20]. CFTR gene with RF can arise from a variety of molecular defects, resulting in sufficient $C F T R$ protein quantity and / or function to allow some ion transport; therefore, $\mathrm{RF}$ mutations are often associated with pancreatic sufficiency and symptoms onset generally occurs at a later age in people with these mutations than in people homozygous for the F508del mutation [21,22]. However, the rate of disease progression in adulthood is similar regardless of CFTR genotype and people with CF who have RF mutations may develop severe lung disease.

In 2020, Salvatore et al. published the results of a compassionate use program of Ivacaftor in 26 Italian CF patients with advanced disease carrying at least one RF mutation. This study showed that treatment with Ivacaftor is safe and results in a clinically significant reduction in antibiotic use and improvement in both lung function and walking distance, even in $\mathrm{CF}$ patients with severe lung disease [23].

Two of the most common RF, $3849+10 \mathrm{~kb} \mathrm{C} \rightarrow \mathrm{T}$ and D1152H, are associated with Ashkenazi Jewish ancestry and are more common in Israel. Recently, Kerem et al. evaluated the effect of two 8-week treatments period of Ivacaftor in 38 people with $\mathrm{CF}$ aged $>6$ years with $3849+10 \mathrm{~kb} \mathrm{C} \rightarrow \mathrm{T}$ or D1152H RF mutations [24]. This placebo-controlled crossover study (NCT03068312) confirmed that Ivacaftor treatment resulted in improvements over 
placebo in lung function measured by Lung Clearance Index (LCI), sweat chloride concentrations, ppFEV1, and CFQ-R respiratory domain score [24].

Effects of Ivacaftor in patients homozygous for the most common mutation (F508del CTFR) were explored in the DISCOVER trial (NCT00953706). After 16 weeks of treatment, no significant change in FEV1, BMI, or CFQ-R score was observed; a small reduction in sweat chloride concentration was reported but not maintained through the next 96 weeks of the study.

In conclusion, Ivacaftor has been shown to affect the life expectancy of CF patients with marked benefits maintained over time, slowing down the respiratory decline, reducing the number of pulmonary exacerbations, and restoring sweat chlorine to normal values. However, Ivacaftor alone is effective only in a small number of CF patients (about 5-8\%). No therapeutic effect was observed for class II variants such as F508del, suggesting that a CFTR potentiator alone cannot be considered as a best therapeutic option for homozygous F508del-CFTR patients. A combination with a corrector is required to facilitate trafficking of the misfolded and prematurely degraded protein to the cell membrane, where a potentiator can rectify the activity defect [25].

\subsection{D9-Ivacaftor (Formerly VX-561 or CTP-656)}

During the 39th European Cystic Fibrosis Conference (June 2016), Vertex Pharmaceuticals submitted results from a phase 1 double-blind placebo-controlled study conducted on a deuterated version of Ivacaftor called VX-561 (formerly CTP-656, D9-Ivacaftor) [26,27]. VX-561 were administered once daily for 7 days and compared to 7 days of Ivacaftor in healthy volunteers. Pharmacokinetic profile of VX-561 was superior concerning half-life, clearance rate, and plasma concentration at $24 \mathrm{~h}$ [26]. These promising data allowed progression to a phase 2 clinical trial (NCT03911713) in subjects older than 18 years in order to assess safety and efficacy in patients with CTFR gating mutation. The study was completed in August 2020, but results are not yet published [27].

\subsection{Icenticaftor (Formerly QBW-251)}

Icenticaftor is a new CTFR potentiator whose safety and efficacy were demonstrated by Kazani et al. in their first-in-human RCT of this molecule (NCT02190604) that included both healthy subjects and CF patients with $\geq 1$ pre-specified CFTR Class III or IV mutation, or homozygous for F508del mutation [28]. Over 14 days of intervention period, Icenticaftor was well tolerated among enrolled individuals with no unexpected events or discontinuations in the CF groups; the most frequent AEs in CF patients were nausea $(12.2 \%)$, headache $(10.2 \%)$, and fatigue (6.1\%). Regarding efficacy, Kazani et al. registered significant improvements in ppFEV1 $(+6.46 \%), \mathrm{LCI} 2.5(-1.13$ points), and sweat chloride $(-8.36 \mathrm{mmol} / \mathrm{L})$ in patients with class III and IV mutations treated with Icenticaftor, while no significant efficacy was observed in patients homozygous for a single F508del. Authors concluded that, similarly to Ivacaftor, monotherapy with Icenticaftor is not clinically sufficient in this subgroup of patients but, based on their results, this molecule might be useful when used in a combination therapeutic with CFTR correctors [28].

\subsection{ABBV-3067 (Formerly GLPG3067)}

ABBV-3067 is a new molecule that acts as a CTFR potentiator. An ongoing phase 2 clinical trial (NCT03969888) is testing its safety and efficacy both alone and in combination with ABBV-2222 (see below) in adult patients homozygous for the F508del mutation; the estimated completion date of the study is fixed in 2022 [29].

\subsection{ABBV-974 (Formerly GLPG1837)}

ABBV-974 (formerly GLPG1837) is a new generation CTFR potentiator, currently in phase 2 clinical trials. SAPHIRA 1 (NCT02707562) is a phase 2 open-label study enrolling subjects with at least one copy of the G551D mutation; SAPHIRA 2 (NCT02690519) is an open-label study testing patients with at least one copy of the S1252N class III mutation [30]. 
Treatment with GLPG1837 was found to be safe and well tolerated in both trials and a statistically significant and dose-dependent decrease in sweat chloride concentration was registered in all groups in SAPHIRA 1 study [30].

\section{CTFR Correctors}

\subsection{Lumacaftor (Formerly VX-809)}

Lumacaftor (formerly VX-809, Figure 4) was the first approved CFTR corrector active on F508del-CFTR protein. Its mechanism of action is yet not completely clear, but several studies indicate that it may repair aberrant assembly of the full-length protein, which is mediated through the NBD1:ICL4 interface, and consequently improves processing, trafficking, and stability of the full-length protein. Therefore, Lumacaftor increases the amount of mature CFTR at the cell surface [31].<smiles>Cc1ccc(NC(=O)C2(c3ccc4c(c3)OC(F)(F)O4)CC2)nc1-c1cccc(C(=O)O)c1</smiles>

Figure 4. Chemical structure of Lumacaftor.

Van Goor et al. studied Lumacaftor's properties in vitro; human bronchial epithelial (HBE) cells from CF lungs incubated for $48 \mathrm{~h}$ with Lumacaftor showed a significant increase in CTFR maturation (eightfold) and in transepithelial chloride transport (fourfold). The same study investigated the combination of Lumacaftor with Ivacaftor: the addition of Ivacaftor to cells homozygous for F508del CTFR further increased the chloride transport, reaching the equivalent to $25 \%$ of non-CF cells [32].

The safety, tolerability, and pharmacodynamics of Lumacaftor as monotherapy was evaluated in adult patients with CF, homozygous for the F508del-CFTR, in a 4-week, double-blind RCT (NCT00865904) [33]. Authors reported reductions in sweat chloride concentration but not statistically significant changes in lung function or patient-reported outcomes. AEs were similar in Lumacaftor and placebo groups. Respiratory events were the most frequently reported and caused discontinuation by one subject in each treatment arm [33].

The mutation F508del produces, as already mentioned, a processing and trafficking defect, resulting in premature degradation, as well as defects in gating and stability for CFTR that localizes to the cell surface [34]. These characteristics provide an explanation as to why Lumacaftor monotherapy was not effective in patients homozygous for this mutation. Moreover, these characteristics indicate that, to better correct the F508del defect, a CFTR potentiator (e.g., Lumacaftor) and corrector (e.g., Ivacaftor) combination should be used.

\subsection{Tezacaftor (Formerly VX-661)}

Tezacaftor (formerly VX-661, Figure 5) is a broad-acting first-generation CFTR corrector designed on the basis of the chemical structure of Lumacaftor yet with improved pharmacokinetics and fewer side effects [31,35]. This small molecule, similarly to Lumacaftor, binds the F508del-CFTR protein and repairs the aberrant ICL:NBD1 interface, facilitating the intracellular processing and trafficking of normal CFTR gene and multiple mutant CFTR forms (including F508del), thereby increasing the amount of CFTR protein at the cell surface and resulting in an enhanced chloride transport [31,35].

In an in vitro study in F/F HBE cells, Tezacaftor in monotherapy and in combination with Ivacaftor was able to improve chloride transport and enhance fluid transport and ciliary beat frequency [36]. 
<smiles>CC(C)(CO)c1cc2cc(NC(=O)C3(c4ccc5c(c4)OC(F)(F)O5)CC3)c(F)cc2n1C[C@H](O)CO</smiles>

Figure 5. Chemical structure of Tezacaftor.

The safety and efficacy of Tezacaftor were evaluated by Donaldson et al. in a randomized, placebo-controlled, double-blind, multicenter, phase 2 study (NCT01531673) in subjects with F/F and F508del/G551D genotypes [35]. According to study design, F/F patients (at least 18 years of age) received Tezacaftor alone or in combination with Ivacaftor. Tezacaftor in monotherapy led to some improvement in lung function in F508del homozygous patients, slightly higher than Lumacaftor [33]. Regarding safety, this study demonstrated a comparable profile across treatment arms with low rates of discontinuation. The majority of AEs among all groups were mild to moderate in nature and the most common were infective pulmonary exacerbations, cough, increased sputum, headache, fatigue, nausea, and diarrhea. The incidence of SAEs was higher in the placebo group, consistently with the higher number of pulmonary exacerbations.

\subsection{Galicaftor (Formerly GLPG2222 or ABBV-2222)}

Galicaftor (formerly GLPG2222) is a novel, potent CFTR corrector restoring the processing and trafficking of the mutated CFTR gene to the plasma membrane. In HBE cells from F/F homozygous subjects, Galicaftor in combination with a potentiator partially restored CFTR function was over 25-fold more potent than Lumacaftor [37]. In 2016, a first human study was conducted to assess safety and pharmacokinetics of ABBV-2222 in healthy adult subjects, reporting promising data [38].

Subsequently, two placebo-controlled phase 2 studies evaluated this molecule in adult F/F patients (FLAMINGO trial, NCT03119649) and in patients heterozygous for F508del CTFR carrying a gating class III mutation, who were receiving Ivacaftor (ALBATROSS trial, NCT03045523) [39]. Results showed a decrease in sweat chloride concentration but no significant changes in pulmonary function or respiratory symptoms; relative to the safety, Galicaftor was well tolerated [39]. Further studies are needed in order to assess its contribution in restoring CTFR function.

\subsection{VX-152, VX-440, VX-445, and VX-659}

VX-152, VX-440, VX-445, and VX-659 are additional next generation CTFR correctors, used in triple combination with Ivacaftor and Tezacaftor (see Section 4).

\subsection{FDL 169}

FDL 169 is a CTFR corrector able to rescue, in F508del HBE cells, CFTR expression with similar efficacy as Lumacaftor. FDL 169 is currently in phase 1-2 trial (NCT03093714, NCT02767297) conducted on subjects aged 18 years and older homozygous for the F508delCFTR mutation, whose results have not yet been published $[40,41]$. 


\section{Double Combination CFTR Modulator Therapy}

\subsection{Lumacaftor + Ivacaftor (ORKAMBI $\left.{ }^{\circledR}\right)$}

Orkambi ${ }^{\circledR}$ was the first approved combination of a CFTR corrector (Lumacaftor) and potentiator (Ivacaftor) by FDA and European Medical Agency (EMA) in 2015. It is indicated in children aged 2 years and older who are homozygous for F508del-CFTR mutation [42]. On one hand, Lumacaftor helps in moving the defective protein to its correct site; on the other hand, Ivacaftor increases the conductance of ions and fluid, powering the protein function.

A phase 2 RCT in patients older than 18 years and a longer (24 weeks) phase 3 RCT in patients older than 12 years (TRAFFIC and TRANSPORT studies, NCT01807923 and NCT01807949, respectively) assessing the safety and efficacy of Lumacaftor/Ivacaftor (LUM/IVA) combination showed slight but significant positive effects, notably on lung function (increase in ppFEV1 3-4\% from baseline) for F508del homozygous patients, together with a significant increase in BMI and a reduced number of exacerbations (30-39\% lower than in placebo) as well as hospitalizations $[43,44]$. The incidence of AEs was generally similar in the LUM/IVA and placebo groups [43,44]. Patients who completed these studies were progressed to 48 weeks in the PROGRESS trial (NCT01931839) and results in the long term were consistent with the TRAFFIC and TRANSPORT trials, confirming the safety and the benefits with a $42 \%$ slower rate of ppFEV1 decline, reduction in the incidence of pulmonary exacerbations, and improvement of BMI in LUM/IVA group [45]. The most common AEs in patients treated with LUM/IVA were infective pulmonary exacerbations, cough, headache, dyspnea, chest tightness, hemoptysis, and increase in sputum production. Four percent of patients treated with LUM/IVA discontinued therapy due to AE versus $1.6 \%$ in the placebo group. Abnormal LFTs and increased blood pressure were also reported but did not lead to severe AEs [44,45].

The aforementioned phase 2 study included a small cohort of F508del heterozygous people who showed no clinical benefit when taking the LUM/IVA combination [43]. This suggests that combination therapy does not provide benefit in F508del heterozygotes unless the second mutation is responsive to Ivacaftor alone (for example, class III mutations). A phase $3 \mathrm{~b}$ open-label prospective study (NCT02390219) evaluating the benefit of LUM/IVA for patients with advanced lung disease (ppFEV1 $<40 \%$ ) observed more frequent respiratory AEs and therefore recommended treatment initiation at a lower dose [46].

Subsequent phase 3 studies of LUM/IVA in children aged 6 to 11 years homozygous for the F508del mutation consistently confirmed a significant improvement in lung function with a decrease in LCI2.5 and increase in FEV1 $[47,48]$. Sweat chloride concentration also decreased significantly, thus providing a CFTR biomarker of modulator efficacy; improvements in nutritional status and health-related quality of life were observed after 24 weeks of treatment. LUM/IVA was well tolerated in this young population. Most common AEs in the LUM/IVA group were productive cough, rhinorrhea, nasal congestion, and abdominal and oropharyngeal pain $[47,48]$.

An additional phase 3, open-label, 24-week study (NCT02797132) assessed the safety, pharmacokinetics, pharmacodynamics, and efficacy of LUM/IVA in children aged $2-5$ years, weighing at least $8 \mathrm{~kg}$ at enrollment, homozygous for F508del-CFTR mutation [49]. Results showed a decrease in the mean sweat chloride concentration, increase in growth parameters, and improvement of biomarkers of pancreatic function (fecal elastase- 1 levels increased and serum immunoreactive trypsinogen levels decreased). Most children (98\%) had at least one AE, but most were mild to moderate in severity. The most common AEs were cough $(63 \%)$, vomiting $(28 \%)$, pyrexia $(28 \%)$, and rhinorrhea $(25 \%)$. Four subjects ( $15 \%$ of patients) experienced more serious events: two cases of infective pulmonary exacerbation, one of constipation, and one of viral gastroenteritis. Discontinuation of treatment was observed in three children, due to elevation of LFTs levels. Authors concluded that LUM/IVA were generally safe and well tolerated in children aged 2-5 years with CF for 24 weeks; efficacy results also highlight the potential of early intervention with LUM/IVA to modify the course of disease [49]. 
Real-life data revealed that clinical response may also vary significantly amongst patients with the same genotype; frequent respiratory AEs and drug intolerance which led to discontinuation of the treatment in some cases were also reported [50]. Notably, interaction between Lumacaftor and Ivacaftor can limit the efficacy of the singular drug. Chronic Ivacaftor exposure reduces Lumacaftor-rescued CFTR in F508del-expressing cells while Lumacaftor reduces plasma concentration of Ivacaftor through the induction of cytochrome CYP3A4 activity; such findings might partially explain the modest efficacy observed in co-treatment with LUM/IVA in clinical trials [51,52].

\subsection{Tezacaftor + Ivacaftor $\left(S Y M D E K O^{\circledR} / S Y M K E V I^{\circledR}\right)$}

A second combination of a CFTR corrector (Tezacaftor) and potentiator (Ivacaftor) was approved in the United States by FDA and in Europe by EMA in 2018 with the names of Symdeko ${ }^{\circledR}$ and Symkevi ${ }^{\circledR}$, respectively $[53,54]$. At present, in Europe, this combination is indicated in subjects aged 6 years and older, homozygous for the F508del mutation or heterozygous for the F508del mutation with one of the following mutations of the CFTR gene: P67L, R117C, L206W, R352Q, A455E, D579G, 711 + 3A $\rightarrow$ G, S945L, S977F, R1070W, $\mathrm{D} 1152 \mathrm{H}, 2789+5 \mathrm{G} \rightarrow \mathrm{A}, 327226 \mathrm{~A} \rightarrow \mathrm{G}$, or $3849+10 \mathrm{kbC} \rightarrow \mathrm{T}$ [53]. In the United States, its use is granted also in subjects with these 12 additional mutations: E56K, R74W, A1067T, E193K, D110H, R347H, D110E, F1052V, F1074L, K1060T, D170N, E831X [54].

Combination therapy with Tezacaftor/Ivacaftor (TEZ/IVA) was evaluated in the EVOLVE trial, a phase 3, randomized, double-blind, multicenter, placebo-controlled, parallel-group study (NCT02347657) in CF patients 12 years of age or older, homozygous for F508del mutation [55]. Through 24 weeks of treatment, significant improvements in mean absolute change in ppFEV1 from baseline (treatment difference $+4.0 \%$ ) as well as in annual estimated rate of pulmonary exacerbations $(-35 \%)$ were observed in the investigational over placebo group. Significant improvements in CFQ-R scores and sweat chloride concentration were also reported in the TEZ/IVA group [55]. No difference was found in BMI between the two therapeutic arms.

Relative to the safety, the incidence of AEs was similar in the two groups, with most of them mild to moderate. Similar results both in terms of efficacy (ppFEV1, sweat chloride concentration) and safety were observed by Donaldson et al. in their randomized, placebocontrolled, double-blind, multicenter, phase 2 study (NCT01531673) conducted in subjects homozygous for F508del and treated with TEZ/IVA for 28 days [36]. Therefore, combination treatment with TEZ/IVA in subjects homozygous for F508del mutation demonstrated comparable therapeutic benefit (in terms of sweat chloride concentration and ppFEV1) to those observed with LUM/IVA in the phase 3 TRAFFIC/TRANSPORT studies [35,55].

Furthermore, the TEZ/IVA combination is characterized by an increased tolerability profile it is because not burdened by the respiratory events (e.g., dyspnea, chest tightness) and the acute lung function decline sometimes registered post LUM/IVA initiation [35,44]. In addition, Tezacaftor is characterized by an ameliorated drug-drug interaction (DDI) profile since it is not a CYP3A4 inducer, thereby interfering with the metabolism of Ivacaftor (substrate of CYP3A4 and CYP3A5) or other drugs (e.g., hormonal contraceptives, Rifampin). On the contrary, Lumacaftor induces (CYP3A, CYP2B6, CYP2C8, CYP2C9, CYP2C19) or inhibits (CYP2C8, CYP2C9) several CYP subtypes [35,44,56,57].

In EXPAND study, the efficacy and safety of Ivacaftor alone or in combination with Tezacaftor were evaluated in CF patients 12 years of age or older heterozygous for F508del mutation and a RF-CFTR mutation [58]. In this phase 3, multicenter, double-blind, threeintervention crossover RCT (NCT02392234), subjects were randomized to receive either combination treatment (TEZ/IVA), monotherapy with Ivacaftor, or matching placebo. After 8 weeks of treatment, in the combination treatment group, the improvement in ppFEV1 ( $+6.8 \%$ vs. placebo) was statistically higher than that observed in the Ivacaftor monotherapy group ( $+4.7 \%$ vs. placebo). Moreover, a significant improvement in absolute change in CFQ-R score was observed, both in TEZ/IVA group and Ivacaftor alone group (11.1 and of 9.7 points, respectively) compared to placebo. Absolute change in sweat 
chloride concentration versus placebo was higher in the combination therapy compared to monotherapy. Relative to the safety, the incidence of AEs was similar across intervention groups with most events (cough, infective pulmonary exacerbations, hemoptysis, and headache) mild or moderate in severity [58].

In following phase 3 clinical trials, co-treatment of TEZ/IVA was demonstrated to reduce sweat chloride concentration and preserve lung function in children aged 6-11 years who are homozygous or heterozygous for the F508del-CFTR mutation and a RF [59]. Such findings served as a basis for the extended approval of TEZ/IVA for patients aged $\geq 6$ years. Extension clinical trials are ongoing to evaluate the longer-term effects of TEZ/IVA (NCT03537651) and the safety and efficacy in younger children [60].

\section{New CFTR Modulators and Triple Combination Therapy}

The evidence of a moderate clinical improvement, notably in respiratory function, obtained with both the dual combinations LUM/IVA and TEZ/IVA in F/F patients and the lack of suitable treatment for minimal function (MF) mutations (that does not produce the protein or produces a protein that is not responsive to Ivacaftor or TEZ/IVA combination), have prompted pharmaceutical companies to develop next-generation correctors, targeting different CFTR sites to maximize the effect of triple-combination treatments [61]. The backbone for this pharmacological approach, known as triple therapy, is represented by TEZ/IVA for its more favorable pharmacological properties, including lower CYP3A activation [62].

Four novel correctors-VX-152, VX-440, VX-445, and VX-659-demonstrated a pronounced improvement of CFTR activity when co-administered with TEZ/IVA in HBE cells (in F/F genotype). In Ussing chamber studies (based on physiological systems used to measure the transport of ions, drugs, and nutrients across epithelial tissues) conducted on HBE cells obtained from F/F homozygous patients, VX-440 and VX-152 in triple combination with Ivacaftor and Tezacaftor restored chloride transport to $\sim 75$ and $65 \%$ of normal CFTR function, respectively [62,63]. In addition, in the same studies using HBE cells collected from CF patients with one copy of F508del and a MF mutation (F/MF), VX-440 and VX-152 in triple combination with Ivacaftor and Tezacaftor restored chloride transport to $\sim 45 \%$ and $40 \%$ of normal CFTR function, respectively [64]. Interestingly, treatment with Ivacaftor/Lumacaftor resulted in chloride transport of $\sim 25 \%$ and $15 \%$ of normal in $\mathrm{HBE}$ cells collected from CF patients with F/F and F/MF genotype, respectively (data shown at 29th NACFC by Vertex Investigator Meeting).

Two phase 2, double-blind RCTs tested safety and efficacy of triple combination of VX440 (NCT02951182) or VX-152 (NCT02951195) with Ivacaftor and Tezacaftor in adult F/MF patients and showed a mean increase in ppFEV1 of $12 \%$ and 9.7\%, respectively [61-64]. Furthermore, F/F patients who had already been treated with Ivacaftor and Tezacaftor experienced a $7.3 \%$ and $9.5 \%$ increase in ppFEV1 after addition of VX-152 or VX-440, respectively. VX-445 (Elexacaftor, Figure 6) and VX-659 (Bamocaftor, Figure 7) were selected for subsequent clinical studies because of their pharmacological properties and long-term safety profiles [61-64].<smiles>Cc1nn(C)cc1S(=O)(=O)NC(=O)c1ccc(-n2ccc(OCC(C)(C)C(F)(F)F)n2)nc1N1C[C@@H](C)CC1(C)C</smiles>

Figure 6. Chemical structure of Elexacaftor. 


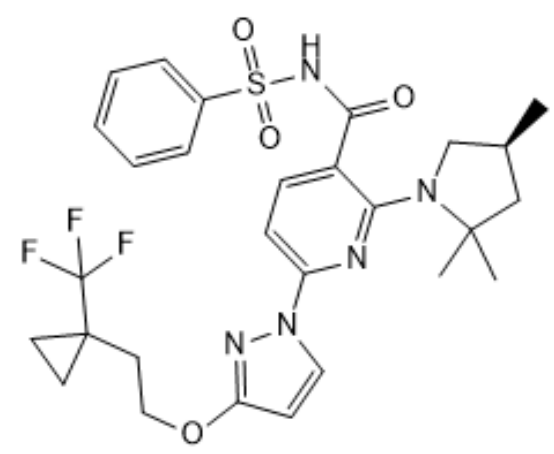

Figure 7. Chemical structure of Bamocaftor.

\subsection{Elexacaftor (Formerly VX-445)}

Elexacaftor (formerly VX-445) was approved in a combination regimen with Tezacaftor and Ivacaftor by the FDA and EMA in October 2019 and in August 2020 under the name of Trikafta ${ }^{\circledR}$ and Kaftrio ${ }^{\circledR}$, respectively $[65,66]$. This therapy is indicated in subjects aged 12 years and older with $\mathrm{F} / \mathrm{F}$ and $\mathrm{F} / \mathrm{MF}$ genotypes (most frequent MF variants: G542X, W1282X, R553X, R1162X, 621 + 1G $\rightarrow$ T, $1717-1 \mathrm{G} \rightarrow \mathrm{A} 1898+1 \mathrm{G} \rightarrow \mathrm{A}$, 3659delC, 394delTT, CFTRdel2,3, N1303K, I507del, G85E, R347P, R560T) [65,66].

The pharmacologic evaluation conducted on HBE cells isolated from CF patients with F/MF and F/F genotypes showed that the combination of Elexacaftor and Tezacaftor, with or without Ivacaftor, increased levels of mature CFTR protein and chloride transport (between $60-80 \%$ of normal CFTR) [67]. These in vitro studies provided the molecular and biologic rationale for investigating the safety and efficacy of Elexacaftor in triple combination with Tezacaftor and Ivacaftor for 4 weeks in a phase 2 double-blind, doseranging RCT (NCT03227471) that involved CF patients 18 years of age or older with $\mathrm{F} / \mathrm{F}$ and $\mathrm{F} / \mathrm{MF}$ genotypes. In this proof-of-concept clinical trial, the triple combination with Elexacaftor/Tezacaftor/Ivacaftor (ELX/TEZ/IVA) significantly increased the ppFEV1 (primary endpoint) in both population groups up to $13.8 \%$ and $11 \%$ in $\mathrm{F} / \mathrm{MF}$ and $\mathrm{F} / \mathrm{F}$ subjects, respectively [67]. This effect was present at the time of the first measurement after the start of treatment ( 2 weeks), indicating that the improvements in lung function occurred rapidly, and was maintained for the entire duration of the study. In both groups, there was also a decrease in sweat chloride concentrations up to $39.1 \mathrm{mmol} / \mathrm{L}$ and an improvement in the respiratory domain of CFQ-R score up to 25.7 points.

Regarding safety, the ELX/TEZ/IVA combination presented an acceptable and comparable AEs profile among patients with both genotypes; $92 \%$ of subjects had an AE, but the majority (97\%) were mild or moderate in severity, and the trial drug was interrupted or discontinued because of an AE event in $8 \%$ of patients [67]. The most common AEs were cough, increased sputum production, hemoptysis, infective pulmonary exacerbation of $\mathrm{CF}$, and fever while no episodes of acute bronchoconstriction were reported. The incidence of laboratory abnormality was $8 \%$ and $3 \%$ for elevation of LFT and bilirubin levels, respectively [67].

The remarkable preliminary results of phase 2 studies on Elexacaftor led to the AURORA program that includes the following studies: VX17-445-102 (NCT03525444) and VX17-445-103 (NCT03525548) to assess safety and efficacy in F/MF and F/F populations, respectively, and VX17-445-105 (NCT03525574), an ongoing 96-week open-label extension study to prosecute randomized placebo-controlled (F/MF) or active-controlled (F/F) treatment studies [62]. Heijerman et al., in their phase 3, multicenter, double-blind RCT (VX17-445-103 Trial, NCT03525548), randomized stable CF patients aged 12 years or older with F/F genotype to receive Elexacaftor-based triple combination or Tezacaftor/Ivacaftor (TEZ/IVA) for 4 weeks [68]. Patients who received ELX/TEZ/IVA combination had a significant increase of 10 points in ppFEV1 than patients who received TEZ/IVA therapy. Likewise, in the triple combination group at week 4 , sweat chloride concentration decreased by $45.1 \mathrm{mmol} / \mathrm{L}$, resulting in a mean value below the diagnostic threshold for $\mathrm{CF}$, and the 
CFQ-R respiratory domain improved by 17.4 points, which exceeds the known 4-point improvement corresponding to the minimal clinically significant difference in patients with CF [68]. Although the treatment duration in this trial was only 4 weeks, an increase in BMI was observed in the ELX/TEZ/IVA group compared with participants who received TEZ/IVA therapy. At trial completion, participants were given the option to enroll in a 96-week open-label extension trial (VX17-445-105; NCT03525574). The triple combination regimen was well tolerated, with no discontinuations. Most AEs were mild or moderate; serious AEs occurred in two (4\%) participants receiving ELX/TEZ/IVA and in one (2\%) receiving TEZ/IVA [68]. The most reported AEs, occurring in more than $10 \%$ of participants in either treatment group, were cough, nasopharyngitis, upper respiratory tract infections, and oropharyngeal pain more often in the ELX/TEZ/IVA arm, and pulmonary exacerbation of CF, hemoptysis, and headache in the TEZ/IVA arm. The authors reported an increase in liver enzymes concentrations in only two (4\%) participants in ELX/TEZ/IVA and in one ( $2 \%)$ participant in the TEZ/IVA group.

Middleton (VX17-445-102, NCT03525444) conducted a wide phase 3 RCT to confirm the efficacy and safety of ELX/TEZ/IVA in $403 \mathrm{CF}$ patients 12 years of age or older with F/MF genotype for 24 weeks [69]. ELX/TEZ/IVA, compared to placebo, resulted in an increase in ppFEV1 of 13.8 points at 4 weeks and 14.3 points through 24 weeks, an increase in CFQ-R respiratory domain score of 20.2 points, a $63 \%$ reduction in rate of pulmonary exacerbations, and a decrease of $41.8 \mathrm{mmol} / \mathrm{L}$ of sweat chloride concentration. BMI also improved significantly at week 24 , with a mean treatment difference of 1.04 relative to placebo. This study also confirmed that ELX/TEZ/IVA was generally safe and had an acceptable side-effect profile. The percentage of patients with at least one AE was $93.1 \%$ in the ELX/TEZ/IVA group and $96.0 \%$ in the placebo group. Most patients had AEs that were mild or moderate and discontinuation of the trial occurred in only 2 patients $(1 \%)$. SAEs occurred in $13.9 \%$ in ELX/TEZ/IVA group and in 20.9\% in placebo group [69]. AEs occurring in at least $10 \%$ of patients in either trial group were consistent with common manifestations and complications of CF: headache, diarrhea, and upper respiratory tract infections were more frequent in triple combination arm, whereas pulmonary exacerbation, cough, sputum increase, hemoptysis, oropharyngeal pain, and fatigue were prevalent in the placebo arm. Elevation of LFTs occurred in $10.9 \%$ of the patients in the ELX/TEZ/IVA group and $4.0 \%$ in the placebo group; furthermore, rash occurred in $10.9 \%$ of the patients in the ELX/TEZ/IVA group and 6.5\% in the placebo group. In both trial groups, rash was more common in female patients than in male patients and more common in female patients who used hormonal contraceptives than in those who did not.

CF patients from both pivotal phase 3 studies $[68,69]$ were invited to participate in an ongoing, phase 3, open-label extension (OLE) study to evaluate the long-term safety and efficacy of ELX/TEZ/IVA [70]. The results of this interim analysis were consistent with pivotal phase 3 studies, demonstrating both the safety and sustained efficacy of long-term (24-36 weeks) ELX/TEZ/IVA treatment in patients CF 12 years or older with one or more F508del allele.

Recently, a prospective observational study including 245 patients aged $\geq 12$ years and with ppFEV1 $<40 \%$ who received ELX/TEZ/IVA for 9 months was conducted in France [71]. In these patients, an absolute increase in ppFEV1 $(+15.1 \%)$ and in weight $(+4.2 \mathrm{~kg})$ were observed; moreover, the number of patients requiring long-term oxygen, non-invasive ventilation, enteral tube feeding, and lung transplantation significantly decreased, suggesting that ELX/TEZ/IVA is associated with rapid clinical improvement also in patients with advanced respiratory disease, often leading them to suspend the waiting list for lung transplantation [71]. Given the strong clinical improvements observed with ELX/TEZ/IVA treatment in CF patients $\geq 12$ years of age, as well as the critical need for more effective CFTR modulation in younger patients with at least one F508del allele, recently, Zemanick et al. evaluated the safety and efficacy of ELX/TEZ/IVA in 66 children of 6-11 years of age with F/MF or F/F genotype in a 24-week open-label, multicentric, phase 3 study (VX18-445-106, NCT03691779) [72]. Through week 24, therapy 
led to improvements consistent with those observed in the controlled phase 3 pivotal studies in older CF patient in AURORA program $[68,69]$ in both genotype cohorts except for sweat chloride concentrations. In this outcome, studied subjects appeared globally more responsive to therapy than older patients but with significative differences between F508del/MF and F508del/F508del genotype: in fact, in the first cohort, the registered sweat chloride decreasing was $-55.1 \mathrm{mmol} / \mathrm{L}$ while in the second one it was $-70.4 \mathrm{mmol} / \mathrm{L}$ from baseline. Regarding respiratory outcomes, ELX/TEZ/IVA improved ppFEV1 and LCI2.5 by 9.1 percentage points and -1.72 units in the F508del/MF cohort, respectively, and by 11.2 percentage points and -1.64 units in the F508del/F508del cohort, respectively [72]. Furthermore, ameliorated CFQ-R scores were reported of a magnitude of 6.9 and 7 points in the F508del/MF and F508del/F508del arms, respectively. Sixty-five children (98.5\%) had AEs, which for most were mild or moderate and generally consistent with manifestations of CF or common childhood infections; the most common were cough, headache, pyrexia, oropharyngeal pain, and upper respiratory tract infection. Only one child experienced a SAE concurrent with respiratory virus infection. Elevation in LFTs was reported in 10.6\% of patients but none of these interrupted or discontinued the study [72]. Rash events occurred in $24.2 \%$ of subjects but all of them were mild or moderate in severity and resolved spontaneously; only one patient discontinued the study because of an erythematous rash that developed after the first dose of ELX/TEZ/IVA. Moreover, for twelve of the children $(75 \%)$, the rash events were assessed as not related to study drug or suggestive of an alternative etiology. To summarize, the study further confirmed the ability of ELX/TEZ/IVA to modulate a single F508del-CFTR allele in patients with CF. The long-term safety and efficacy of ELX/TEZ/IVA in CF children who are 6 years of age and older will be assessed in the ongoing 96-week open-label extension study (VX19-445-107, NCT04183790) that enrolled all 64 patients who completed the treatment period of Zemanick et al.'s study [72].

In conclusion, the magnitude of therapeutic responses obtained with ELX/TEZ/IVA triple combination was greater than that achieved with benchmark Ivacaftor in patients with G551D [13,35] or other gating mutations [15], justifying the term of Highly Effective Modulator Treatment (HEMT).

\subsection{Bamocaftor (Formerly VX-659)}

In Ussing chamber studies, Bamocaftor in triple combination with Ivacaftor/Tezacaftor restored chloride transport to $\sim 65 \%$ of normal CFTR in HBE cells collected from F/MF patients [73]. Similarly to Elexacaftor, after in vitro pharmacologic and biochemical studies on HBE from CF patients with F/MF or F/F genotypes, Bamocaftor in triple combination with TEZ/IVA was tested in a randomized, placebo-controlled, double-blind, multicenter, phase 1 trial (NCT 03029455, VX-16-659-001) conducted to evaluate preliminary pharmacokinetics and initial safety in a small group of healthy volunteers and CF patients. Most AEs were mild or moderate, and none led to interruption of the trial regimen [74].

Subsequently, the safety and efficacy of this molecule were evaluated in a larger, randomized, parallel-track, placebo- or active-controlled, double-blind, multicenter, doseranging, phase 2 trial (VX-16-659-101, NCT03224351) enrolling 117 CF patients 18 years of age or older with F/F and F/MF genotype [74]. In this study, Bamocaftor in triple combination therapy showed an acceptable safety profile in both cohorts. The majority of AEs were mild to moderate and the most common were cough, infective pulmonary exacerbation, oropharyngeal pain, headache, and increased sputum production. No episodes of acute bronchoconstriction were reported. Two cases of elevation of LFT were reported in patients who received Bamocaftor/Tezacaftor/Ivacaftor (VX-659/TEZ/IVA) without concurrent elevation in the bilirubin level.

Regarding efficacy, triple combination with VX-659/TEZ/IVA produced at 4 weeks a significant increase in ppFEV1 up to 13.3 and 9.7 percentage points over the comparator in F/MF and F/F cohorts, respectively. Among F/MF and F/F cohorts, respective improvements in absolute change in sweat chloride concentration and CFQ-R scores were up to 
$-51.4 \mathrm{mmol} / \mathrm{L}$ and $-42.2 \mathrm{mmol} / \mathrm{L}$ and up to +24.6 and +19.5 points over the comparator in favor of triple combination therapy arm [74].

The robust results of phase 2 studies on Bamocaftor led to the advancement to phase 3 studies (ECLIPSE programs). The ECLIPSE program includes VX17-659-102 (NCT03447249) and VX17-659-103 (NCT03460990) to assess safety and efficacy in F/MF and F/F cohorts, respectively, and VX17-659-105 (NCT03447262) for the ongoing 96-week open-label extension study of the program [62]. A phase 3, randomized, double-blind, controlled study (VX17-659-103 trial) evaluating the efficacy and safety of Bamocaftor-based triple therapy in $116 \mathrm{CF}$ patients with $\mathrm{F} / \mathrm{F}$ genotype demonstrated their superiority to TEZ/IVA at week 4 both for efficacy and safety [75]. Regarding efficacy, ppFEV1 from baseline at week 4 increased significantly by 9.9 percentage points and sweat chloride concentration decreased significantly by $-48.7 \mathrm{mmol} / \mathrm{L}$, under the diagnostic threshold for $\mathrm{CF}$, in the Bamocaftor triple therapy group; in addition, CFQ-R significantly improved by 13.5 points in this cohort. In VX17-659-103 trials, Bamocaftor triple combination therapy demonstrated its safety and tolerability: the percentage of patients with at least one $\mathrm{AE}$ was $61.1 \%$ in the VX-659/TEZ/IVA group and $54.4 \%$ in the TEZ/IVA group [75]. The most frequent AEs were sputum increase, cough, diarrhea in Bamocaftor triple therapy, and infective pulmonary exacerbation and headache in the TEZ/IVA group. Serious AEs were reported in two patients (4\%) in VX-659/TEZ/IVA arm (constipation in one patient and depression with suicidal ideation in another), whereas no SAEs occurred in the TEZ/IVA arm; no deaths were registered in either trial group.

A second phase 3, large, randomized, double-blind RCT (VX17-659-102) was conducted to assess the efficacy and safety of 24 weeks of VX-659/TEZ/IVA combination therapy in 385 subjects with CF older than 12 years and with F/MF genotype [76]. Bamocaftor combination therapy produced significant benefits in term of lung function also in F/MF subjects; it induced significant improvement in ppFEV1 of 14.0 and 14.2 percentage points at week 4 and at week 24, respectively. From the patient point of view, CFQ-R scores improved by 17.9 and 20.1 points at week 4 and week 24 , respectively, for VX-659 based therapy [76]. In addition, at week 24, with Bamocaftor triple therapy, the number of pulmonary exacerbations and the sweat chloride concentration were significantly reduced versus placebo by $14 \%$ and $-44.6 \mathrm{mmol} / \mathrm{L}$, respectively. The nutritional status also improved significantly, with an increase in BMI of 1.11 with VX-659/TEZ/IVA over the placebo. Regarding safety, no difference was found in the percentage of patients with at least one AE between VX-659/TEZ/IVA combination therapy (90\%) and placebo (93\%) [76]. In the VX17-659-102 trial, AEs were almost mild to moderate and the most reported of them were upper respiratory tract infection, cough, sputum increase, nasopharyngitis, and headache in the Bamocaftor group and infective pulmonary exacerbation, cough, sputum increase, and headache in the placebo group [76]. The serious AEs were less frequent in the investigational group (6\%) compared to placebo group (31\%). There were no deaths in either trial group.

\subsection{Proteostasis Pipeline}

Proteostasis Therapeutics Inc. (PTI) developed a triple combination with a potentiator (PTI-808 or Dirocaftor), a corrector (PTI-801 or Posenacaftor), and an amplifier. The amplifier PTI-428 (or Nesolicaftor) demonstrated to increase the production of defective CFTR mRNA in vitro for many mutations, including F508del and some rare mutations without increased expression of the CFTR at the plasma membrane proving that CFTR remained dysfunctional $[77,78]$. A phase $1 / 2$ clinical study (NCT03500263) in CF adults homozygous for F508del showed an improvement of $8 \%$ in ppFEV1 and a decrease of $29 \mathrm{mmol} / \mathrm{L}$ in sweat chloride after 4 weeks of treatment compared to the placebo dysfunctional [79]. 


\section{Conclusions}

CFTR modulators represent a turning point in the therapeutic approach to CF and have radically changed its nature by evolving from being an option for a niche of $\mathrm{CF}$ patients to representing a key therapeutic option beyond all expectations for the majority of CF patients, with increasingly promising results. Table 1 shows approved CFTR modulators with their indications, whereas Table 2 summarizes clinical trials on CFTR-modulators triple therapy.

Table 1. Approved CFTR modulators and their indications.

\begin{tabular}{|c|c|c|c|c|}
\hline Modulators & $\begin{array}{l}\text { Commercial } \\
\text { Name }\end{array}$ & Approval Year & Responsive Mutations & $\begin{array}{l}\text { Approved } \\
\text { Ages }\end{array}$ \\
\hline Ivacaftor & $\begin{array}{l}\text { Kalydeco }^{\circledR} \\
\text { (EU/USA) }\end{array}$ & 2012 & $\begin{array}{l}\text { G551D, S549N, G1244E, G178R, S1251N, G551S, } \\
\text { G1349D, S1255P, R117H, E56K, K1060T, P67L, } \\
\text { E193K, A1067T, R74W, L206W, G1069R, D110E, } \\
\text { R347H, D579G, R1070Q, D1270N, D110H, R352Q, } \\
\text { S945L, R1070W, R117C, A455E, S977F, F1074L, } \\
\text { F1052V, D115H; 3849+10 kb C>T, 2789+5G>A, } \\
\text { 3273-26A>G, 711+3A>G, E831X }\end{array}$ & $\geq 4$ months \\
\hline $\begin{array}{l}\text { Lumacaftor- } \\
\text { Ivacaftor }\end{array}$ & $\begin{array}{l}\text { Orkambi }^{\circledR} \\
\text { (EU/USA) }\end{array}$ & 2015 & Two copy of F508del & $\geq 2$ years \\
\hline $\begin{array}{l}\text { Tezacaftor- } \\
\text { Ivacaftor }\end{array}$ & $\begin{array}{c}\text { Symkevi }^{\circledR}(\mathrm{EU}) \\
\text { Symdeko }^{\circledR} \text { (USA) }\end{array}$ & 2018 & $\begin{array}{c}\text { Two copy of F508del } \\
\text { One copy of F508del in association with E56K, } \\
\text { K1060T, P67L, E193K, A1067T, R74W, L206W, } \\
\text { D110E, D110H, R347H, D579G, R1070Q, D1270N, } \\
\text { R352Q, S945L, R1070W, R117C, A455E, S977F, } \\
\text { F1074L, F1052V, D1152H, 3849+10 kb C>T, } \\
\text { 2789+5G>A, 327326A>G, 711+3A>G }\end{array}$ & $\geq 6$ years \\
\hline $\begin{array}{l}\text { Elexacaftor- } \\
\text { Tezacaftor- } \\
\text { Ivacaftor }\end{array}$ & $\begin{array}{c}\text { Kaftrio }^{\circledR}(\mathrm{EU}) \\
\text { Trikafta }^{\circledR} \text { (USA) }\end{array}$ & $\begin{array}{c}2020 \text { (EU) } \\
2019 \text { (USA) }\end{array}$ & One copy of F508del & $\geq 12$ years \\
\hline
\end{tabular}

Table 2. Summary of clinical trials on CFTR-modulators triple therapy. Abbreviations: F/F = F508del/F508del genotype, $\mathrm{F} / \mathrm{MF}=\mathrm{F} 508 \mathrm{del} / \mathrm{MF}$ genotype.

\begin{tabular}{|c|c|c|c|c|c|c|c|c|c|c|}
\hline \multicolumn{5}{|c|}{ Study } & \multicolumn{3}{|c|}{ Patients Characteristics } & \multicolumn{3}{|c|}{ Outcomes } \\
\hline $\begin{array}{l}\text { Study } \\
\text { Identifier } \\
\text { (NCT) }\end{array}$ & Name & $\begin{array}{l}\text { First Author, } \\
\text { Year }\end{array}$ & Phase & $\begin{array}{l}\text { Investigational } \\
\text { Drug }\end{array}$ & $\begin{array}{c}\text { Age } \\
\text { (Years) }\end{array}$ & Genotype & $\begin{array}{l}\text { Number of } \\
\text { Patients }\end{array}$ & $\begin{array}{c}\text { ppFEV1 } \\
(\%)\end{array}$ & $\begin{array}{c}\text { Sweat } \\
\text { Chloride } \\
\text { (mmol/L) }\end{array}$ & $\begin{array}{c}\text { CFQ-R } \\
\text { Score } \\
\text { (Points) }\end{array}$ \\
\hline \multirow{2}{*}{ NCT03227471 } & \multirow{2}{*}{$\begin{array}{l}\text { VX16-445- } \\
001\end{array}$} & \multirow{2}{*}{ Keating, 2018} & \multirow{2}{*}{2} & \multirow{2}{*}{ ELX/TEZ/IVA } & \multirow{2}{*}{$\geq 18$} & F508del/F508del & 28 & $+11\left({ }^{*}\right)$ & $-39.6\left(^{*}\right)$ & $+20.7(*)$ \\
\hline & & & & & & F508del/MF & 95 & $+13.8\left(^{*}\right)$ & $-39.1\left(^{*}\right)$ & $+25.7(*)$ \\
\hline \multirow{2}{*}{ NCT03224351 } & \multirow{2}{*}{$\begin{array}{l}\text { VX16-659- } \\
101\end{array}$} & \multirow{2}{*}{ Davies, 2018} & \multirow{2}{*}{2} & \multirow{2}{*}{$\begin{array}{c}\text { VX- } \\
\text { 659/TEZ/IVA }\end{array}$} & \multirow{2}{*}{$\geq 18$} & F508del/F508del & 29 & $+9.7(*)$ & $-42.2(*)$ & $+19.5\left(^{*}\right)$ \\
\hline & & & & & & F508del/MF & 88 & $+13.3\left(^{*}\right)$ & $-51.4\left(^{*}\right)$ & $+24.6\left(^{*}\right)$ \\
\hline NCT03525548 & $\begin{array}{c}\text { VX17-445- } \\
103\end{array}$ & $\begin{array}{l}\text { Heijerman, } \\
2019\end{array}$ & 3 & ELX/TEZ/IVA & $\geq 12$ & F508del/F508del & 107 & $+10\left(^{*}\right)$ & $-45.1\left(^{*}\right)$ & $+17.4\left(^{*}\right)$ \\
\hline NCT03525444 & $\begin{array}{l}\text { VX17-445- } \\
102\end{array}$ & $\begin{array}{l}\text { Middleton, } \\
2019\end{array}$ & 3 & ELX/TEZ/IVA & $\geq 12$ & F508del/MF & 403 & $+13.8\left(^{*}\right)$ & $-41.8(* *)$ & $+20.2(* *)$ \\
\hline NCT03460990 & $\begin{array}{c}\text { VX17-659- } \\
103\end{array}$ & - & 3 & $\begin{array}{c}\text { VX- } \\
\text { 659/TEZ/IVA }\end{array}$ & $\geq 12$ & F508del/F508del & 111 & $+9.9(*)$ & $-48.7\left(^{*}\right)$ & $+13.5\left(^{*}\right)$ \\
\hline NCT03447249 & $\begin{array}{l}\text { VX17-659- } \\
102\end{array}$ & - & 3 & $\begin{array}{c}\text { VX- } \\
\text { 659/TEZ/IVA }\end{array}$ & $\geq 12$ & F508del/MF & 382 & $+14\left(^{*}\right)$ & $-44.6\left(^{(* *}\right)$ & $+20.1(* *)$ \\
\hline \multirow{2}{*}{ NCT03691779 } & \multirow{2}{*}{$\begin{array}{l}\text { VX18-445- } \\
\quad 106\end{array}$} & \multirow{2}{*}{$\begin{array}{l}\text { Zemanick, } \\
2021\end{array}$} & \multirow{2}{*}{3} & \multirow{2}{*}{ ELX/TEZ/IVA } & \multirow{2}{*}{$6-11$} & F508del/F508del & 29 & $+11.2\left({ }^{* *}\right)$ & $-70.4\left(^{* *}\right)$ & $+7(* *)$ \\
\hline & & & & & & F508del/MF & 37 & $+9.1(* *)$ & $-55.1\left(^{* *}\right)$ & $+6.9(* *)$ \\
\hline
\end{tabular}

$\left.{ }^{*}\right)$ : Outcome through week 4. $\left(^{* *}\right)$ : Outcome through week 24. 
Based on the known impact of the benchmark therapy Ivacaftor in a small subset of $\mathrm{CF}$ patients, the new combinations have been shown to change profoundly the clinical course of $\mathrm{CF}$, leading to meaningful improvements in the lives of such a large proportion of people with CF heterozygous for F508del, especially if started in young children. Further studies are needed to extend the use of triple CFTR modulation therapy also for young children in order to prevent the irreversible effects of the disease and to provide patients with very rare mutations with a personalized approach to treatment. In addition, further studies could allow us to understand the efficacy and safety of new drugs in development (i.e., ABBV3067 and ABBV191 from Abbvie, ELX-02 from Eloxx Pharmaceuticals) as well as their synergy with the available molecules.

Author Contributions: A.M. wrote the first draft of the manuscript; V.F. co-wrote the manuscript and gave a substantial scientific contribution; M.D., G.C. and F.M. performed the literature review; C.C. supervised the literature analysis; S.E. and G.P. supervised the project, revised the text, and gave a substantial scientific contribution. All authors have read and agreed to the published version of the manuscript.

Funding: This manuscript was supported by the Cystic Fibrosis Center of Emilia-Romagna Region, University Hospital of Parma, Parma, Italy.

Institutional Review Board Statement: Not applicable.

Informed Consent Statement: Not applicable for a review.

Data Availability Statement: Data sharing not applicable. The text has been written on findings reported in the mentioned References.

Conflicts of Interest: The authors declare no conflict of interest.

\section{References}

1. Connett, G.J. Lumacaftor-ivacaftor in the treatment of cystic fibrosis: Design, development and place in therapy. Drug Des. Dev. Ther. 2019, 13, 2405-2412. [CrossRef] [PubMed]

2. Saint-Criq, V.; Gray, M.A. Role of CFTR in epithelial physiology. Cell. Mol. Life Sci. 2017, 74, 93-115. [CrossRef] [PubMed]

3. Sharma, J.; Keeling, K.M.; Rowe, S.M. Pharmacological approaches for targeting cystic fibrosis nonsense mutations. Eur. J. Med. Chem. 2020, 200, 112436. [CrossRef]

4. Rafeeq, M.M.; Murad, H.A.S. Cystic fibrosis: Current therapeutic targets and future approaches. J. Transl. Med. 2017, 15, 84. [CrossRef]

5. De Boeck, K. Cystic fibrosis in the year 2020: A disease with a new face. Acta Paediatr. 2020, 109, 893-899. [CrossRef]

6. McKone, E.F.; Emerson, S.S.; Edwards, K.L.; Aitken, M.L. Effect of genotype on phenotype and mortality in cystic fibrosis: A retrospective cohort study. Lancet 2003, 361, 1671-1676. [CrossRef]

7. Cystic Fibrosis Trust. UK Cystic Fibrosis Registry Annual Data Report 2019. Available online: https://www.cysticfibrosis.org.uk/ sites / default/ files/2020-12/2019\%20Registry\%20Annual\%20Data\%20report_Sep\%202020.pdf (accessed on 14 April 2021).

8. Quon, B.S.; Rowe, S.M. New and emerging targeted therapies for cystic fibrosis. BMJ 2016, 352, i859. [CrossRef]

9. Harman, K.; Dobra, R.; Davies, J.C. Disease-modifying drug therapy in cystic fibrosis. Paediatr. Respir. Rev. 2018, 26, 7-9. [CrossRef]

10. Eckford, P.D.; Li, C.; Ramjeesingh, M.; Bear, C.E. Cystic fibrosis transmembrane conductance regulator (CFTR) potentiator VX-770 (ivacaftor) opens the defective channel gate of mutant CFTR in a phosphorylation-dependent but ATP-independent manner. J. Biol. Chem. 2012, 287, 36639-36649. [CrossRef] [PubMed]

11. Harutyunyan, M.; Huang, Y.; Mun, K.S.; Yang, F.; Arora, K.; Naren, A.P. Personalized medicine in CF: From modulator development to therapy for cystic fibrosis patients with rare CFTR mutations. Am. J. Physiol. Lung Cell. Mol. Physiol. 2018, 314, L529-L543. [CrossRef]

12. European Medicines Agency. Kalydeco. Available online: https://www.ema.europa.eu/en/medicines/human/EPAR/kalydeco (accessed on 1 September 2020).

13. Ramsey, B.W.; Davies, J.; McElvaney, N.G.; Tullis, E.; Bell, S.C.; Dřevínek, P.; Griese, M.; McKone, E.F.; Wainwright, C.E.; Konstan, M.W.; et al. A CFTR potentiator in patients with cystic fibrosis and the G551D mutation. N. Engl. J. Med. 2011, 365, 1663-1672. [CrossRef] [PubMed]

14. Davies, J.C.; Wainwright, C.E.; Canny, G.J.; Chilvers, M.A.; Howenstine, M.S.; Munck, A.; Mainz, J.G.; Rodriguez, S.; Li, H.; Yen, K.; et al. Efficacy and safety of ivacaftor in patients aged 6 to 11 years with cystic fibrosis with a G551D mutation. Am. J. Respir. Crit. Care Med. 2013, 187, 1219-1225. [CrossRef] [PubMed] 
15. De Boeck, K.; Munck, A.; Walker, S.; Faro, A.; Hiatt, P.; Gilmartin, G.; Higgins, M. Efficacy and safety of ivacaftor in patients with cystic fibrosis and a non-G551D gating mutation. J. Cyst. Fibros. 2014, 13, 674-680. [CrossRef] [PubMed]

16. Moss, R.B.; Flume, P.A.; Elborn, J.S.; Cooke, J.; Rowe, S.M.; McColley, S.A.; Rubenstein, R.C.; Higgins, M.; VX11-770-110 (KONDUCT) Study Group. Efficacy and safety of ivacaftor in patients with cystic fibrosis who have an Arg117His-CFTR mutation: A double-blind, randomised controlled trial. Lancet Respir. Med. 2015, 3, 524-533. [CrossRef]

17. Davies, J.C.; Robertson, S.; Green, Y.; Rosenfeld, M. An open-label study of the safety, pharmacokinetics, and pharmacodynamics of ivacaftor in patients aged 2 to 5 years with CF and CFTR gating mutation: The KIWI study. In Proceedings of the 28th Annual North American Conference of the Cystic Fibrosis Foundation, Atlanta, GA, USA, 9-11 October 2014.

18. Rosenfeld, M.; Cunningham, S.; Harris, W.T.; Lapey, A.; Regelmann, W.E.; Sawicki, G.S.; Southern, K.W.; Chilvers, M.; Higgins, M.; Tian, S.; et al. An open-label extension study of ivacaftor in children with CF and a CFTR gating mutation initiating treatment at age 2-5 years (KLIMB). J. Cyst. Fibros. 2019, 18, 838-843. [CrossRef]

19. Rosenfeld, M.; Wainwright, C.E.; Higgins, M.; Wang, L.T.; McKee, C.; Campbell, D.; Tian, S.; Schneider, J.; Cunningham, S.; Davies, J.C.; et al. Ivacaftor treatment of cystic fibrosis in children aged 12 to $<24$ months and with a CFTR gating mutation (ARRIVAL): A phase 3 single-arm study. Lancet Respir. Med. 2018, 6, 545-553, Erratum in: Lancet Respir. Med. 2018, 6, e35. Erratum in: Lancet Respir. Med. 2019, 7, e15.. [CrossRef]

20. Durmowicz, A.G.; Lim, R.; Rogers, H.; Rosebraugh, C.J.; Chowdhury, B.A. The U.S. Food and Drug Administration's Experience with Ivacaftor in Cystic Fibrosis. Establishing Efficacy Using In Vitro Data in Lieu of a Clinical Trial. Ann. Am. Thorac. Soc. 2018, 15, 1-2. [CrossRef]

21. Van Goor, F.; Yu, H.; Burton, B.; Hoffman, B.J. Effect of ivacaftor on CFTR forms with missense mutations associated with defects in protein processing or function. J. Cyst. Fibros. 2014, 13, 29-36. [CrossRef] [PubMed]

22. Duguépéroux, I.; De Braekeleer, M. The CFTR 3849+10kbC->T and 2789+5G->A alleles are associated with a mild CF phenotype. Eur. Respir. J. 2005, 25, 468-473. [CrossRef]

23. Salvatore, D.; Terlizzi, V.; Francalanci, M.; Taccetti, G.; Messore, B.; Biglia, C.; Pisi, G.; Calderazzo, M.A.; Caloiero, M.; Pizzamiglio, G.; et al. Ivacaftor improves lung disease in patients with advanced CF carrying CFTR mutations that confer residual function. Respir. Med. 2020, 171, 106073. [CrossRef]

24. Kerem, E.; Cohen-Cymberknoh, M.; Tsabari, R.; Wilschanski, M.; Reiter, J.; Shoseyov, D.; Gileles-Hillel, A.; Pugatsch, T.; Davies, J.C.; Short, C.; et al. Ivacaftor in People with Cystic Fibrosis and a 3849+10kb C $\rightarrow$ T or D1152H Residual Function Mutation. Ann. Am. Thorac. Soc. 2021, 18, 433-441. [CrossRef]

25. Flume, P.A.; Liou, T.G.; Borowitz, D.S.; Li, H.; Yen, K.; Ordoñez, C.L.; Geller, D.E.; VX 08-770-104 Study Group. Ivacaftor in subjects with cystic fibrosis who are homozygous for the F508del-CFTR mutation. Chest 2012, 142, 718-724. [CrossRef]

26. Uttamsingh, V.; Pilja, L.; Brummel, C.L.; Grotbeck, B.; Cassella, J.V.; Braman, G. Ctp-656 Multiple Dose Pharmacokinetic Profile Continues to Support a Once-Daily Potentiator for Cystic Fibrosis Patients with Gating Mutations. In Proceedings of the 30th Annual North American Cystic Fibrosis Conference Cystic Fibrosis Foundation, Orlando, FL, USA, 27-29 October 2016.

27. Vertex Pharmaceuticals Incorporated. A Phase 2 Study to Evaluate Efficacy and Safety of VX-561 in Subjects Aged 18 Years and Older with Cystic Fibrosis. NLM Identifier: NCT03911713. Available online: https:/ / clinicaltrials.gov/ct2/show/NCT03911713 (accessed on 23 September 2020).

28. Kazani, S.; Rowlands, D.J.; Bottoli, I.; Milojevic, J.; Alcantara, J.; Jones, I.; Kulmatycki, K.; Machineni, S.; Mostovy, L.; Nicholls, I.; et al. Safety and efficacy of the cystic fibrosis transmembrane conductance regulator potentiator icenticaftor (QBW251). J. Cyst. Fibros. 2021, 20, 250-256. [CrossRef]

29. AbbVie. A Phase 2 Study of ABBV-3067 alone and in Combination with ABBV-2222. NLM Identifier: NCT03969888. Available online: https:/ / clinicaltrials.gov/ct2/show / NCT03969888 (accessed on 23 September 2020).

30. Conrath, K.; Gesson, C.; Allamassey, L.; Van de Steen, O.; Kanters, D.; De Kock, H.; De Boek, C.; Davies, J.C. Glpg1837 in subjects with cystic fibrosis and the S1251n or G551d mutation: Results from phase 2a studies (Saphira 1 and 2). In Proceedings of the 31st Annual North American Cystic Fibrosis Conference Cystic Fibrosis Foundation, Indianapolis, IN, USA, 2-4 November 2017.

31. Guerra, L.; Favia, M.; Di Gioia, S.; Laselva, O.; Bisogno, A.; Casavola, V.; Colombo, C.; Conese, M. The preclinical discovery and development of the combination of ivacaftor + tezacaftor used to treat cystic fibrosis. Expert Opin. Drug Discov. 2020, 15, 873-891. [CrossRef] [PubMed]

32. Van Goor, F.; Hadida, S.; Grootenhuis, P.D.J.; Burton, B.; Stack, J.H.; Straley, K.S.; Decker, C.J.; Miller, M.; McCartney, J.; Olson, E.R.; et al. Correction of the F508del-CFTR protein processing defect in vitro by the investigational drug VX-809. Proc. Natl. Acad. Sci. USA 2011, 108, 18843-18848. [CrossRef]

33. Clancy, J.P.; Rowe, S.M.; Accurso, F.J.; Aitken, M.L.; Amin, R.S.; Ashlock, M.A.; Ballmann, M.; Boyle, M.P.; Bronsveld, I.; Campbell, P.W.; et al. Results of a phase IIa study of VX-809, an investigational CFTR corrector compound, in subjects with cystic fibrosis homozygous for the F508del-CFTR mutation. Thorax 2012, 67, 12-18. [CrossRef] [PubMed]

34. Phuan, P.W.; Yang, B.; Knapp, J.M.; Wood, A.B.; Lukacs, G.L.; Kurth, M.J.; Verkman, A.S. Cyanoquinolines with independent corrector and potentiator activities restore $\triangle P$ he508-cystic fibrosis transmembrane conductance regulator chloride channel function in cystic fibrosis. Mol. Pharmacol. 2011, 80, 683-693. [CrossRef] [PubMed]

35. Donaldson, S.H.; Pilewski, J.M.; Griese, M.; Cooke, J.; Viswanathan, L.; Tullis, E.; Davies, J.C.; Lekstrom-Himes, J.A.; Wang, L.T.; VX11-661-101 Study Group. Tezacaftor/Ivacaftor in Subjects with Cystic Fibrosis and F508del/F508del-CFTR or F508del/G551DCFTR. Am. J. Respir. Crit. Care Med. 2018, 197, 214-224. [CrossRef] 
36. Shiferaw, D.; Faruqi, S. Profile of tezacaftor/ivacaftor combination and its potential in the treatment of cystic fibrosis. Ther. Clin. Risk Manag. 2019, 15, 1029-1040, Erratum in: Ther. Clin. Risk Manag. 2019, 15, 1207. [CrossRef]

37. Wang, X.; Liu, B.; Searle, X.; Yeung, C.; Bogdan, A.; Greszler, S.; Singh, A.; Fan, Y.; Swensen, A.M.; Vortherms, T.; et al. Discovery of 4-[(2R,4R)-4-(\{[1-(2,2-Difluoro-1,3-benzodioxol-5-yl)cyclopropyl]carbonyl\}amino)-7-(difluoromethoxy)-3,4-dihydro2H-chromen-2-yl]benzoic Acid (ABBV/GLPG-2222), a Potent Cystic Fibrosis Transmembrane Conductance Regulator (CFTR) Corrector for the Treatment of Cystic Fibrosis. J. Med. Chem. 2018, 61, 1436-1449. [CrossRef]

38. Galapagos, N.V. First-in-Human Single and Multiple Dose of GLPG2222. NLM Identifier: NCT02662452. Available online: https: / / clinicaltrials.gov/ct2/show / NCT02662452 (accessed on 29 September 2020).

39. Bell, S.C.; Barry, P.J.; De Boeck, K.; Drevinek, P.; Elborn, J.S.; Plant, B.J.; Minić, P.; Van Braeckel, E.; Verhulst, S.; Muller, K.; et al. CFTR activity is enhanced by the novel corrector GLPG2222, given with and without ivacaftor in two randomized trials. J. Cyst. Fibros. 2019, 18, 700-707. [CrossRef]

40. Flatley Discovery Lab LLC. A Study to Evaluate Safety, PK and PD of FDL169 in Cystic Fibrosis Subjects. NLM Identifier: NCT03093714. Available online: https:/ / clinicaltrials.gov/ct2/show /NCT03093714 (accessed on 30 September 2020).

41. Flatley Discovery Lab LLC. Bioavailability and Pharmacokinetics Study of FDL169 in Healthy Subjects and Subjects with Cystic Fibrosis. NLM Identifier: NCT02767297. Available online: https:/ / clinicaltrials.gov/ct2/show/NCT02767297 (accessed on 30 September 2020).

42. European Medicines Agency. Orkambi. Available online: https://www.ema.europa.eu/en/medicines/human/EPAR/orkambi (accessed on 1 September 2020).

43. Boyle, M.P.; Bell, S.C.; Konstan, M.W.; McColley, S.A.; Rowe, S.M.; Rietschel, E.; Huang, X.; Waltz, D.; Patel, N.R.; Rodman, D.; et al. A CFTR corrector (lumacaftor) and a CFTR potentiator (ivacaftor) for treatment of patients with cystic fibrosis who have a phe508del CFTR mutation: A phase 2 randomised controlled trial. Lancet Respir. Med. 2014, 2, 527-538. [CrossRef]

44. Wainwright, C.E.; Elborn, J.S.; Ramsey, B.W.; Marigowda, G.; Huang, X.; Cipolli, M.; Colombo, C.; Davies, J.C.; De Boeck, K.; Flume, P.A.; et al. Lumacaftor-Ivacaftor in Patients with Cystic Fibrosis Homozygous for Phe508del CFTR. N. Engl. J. Med. 2015, 373, 220-231. [CrossRef]

45. Konstan, M.W.; McKone, E.F.; Moss, R.B.; Marigowda, G.; Tian, S.; Waltz, D.; Huang, X.; Lubarsky, B.; Rubin, J.; Millar, S.J.; et al. Assessment of safety and efficacy of long-term treatment with combination lumacaftor and ivacaftor therapy in patients with cystic fibrosis homozygous for the F508del-CFTR mutation (PROGRESS): A phase 3, extension study. Lancet Respir. Med. 2017, 5, 107-118. [CrossRef]

46. Taylor-Cousar, J.; Niknian, M.; Gilmartin, G.; Pilewski, J.M.; VX11-770-901 Investigators. Effect of ivacaftor in patients with advanced cystic fibrosis and a G551D-CFTR mutation: Safety and efficacy in an expanded access program in the United States. J. Cyst. Fibros. 2016, 15, 116-122. [CrossRef]

47. Ratjen, F.; Hug, C.; Marigowda, G.; Tian, S.; Huang, X.; Stanojevic, S.; Milla, C.E.; Robinson, P.D.; Waltz, D.; Davies, J.C.; et al. Efficacy and safety of lumacaftor and ivacaftor in patients aged 6-11 years with cystic fibrosis homozygous for F508del-CFTR: A randomised, placebo-controlled phase 3 trial. Lancet Respir. Med. 2017, 5, 557-567. [CrossRef]

48. Milla, C.E.; Ratjen, F.; Marigowda, G.; Liu, F.; Waltz, D.; Rosenfeld, M.; VX13-809-011 Part B Investigator Group. Lumacaftor/Ivacaftor in Patients Aged 6-11 Years with Cystic Fibrosis and Homozygous for F508del-CFTR. Am. J. Respir. Crit. Care Med. 2017, 195, 912-920. [CrossRef]

49. McNamara, J.J.; McColley, S.A.; Marigowda, G.; Liu, F.; Tian, S.; Owen, C.A.; Stiles, D.; Li, C.; Waltz, D.; Wang, L.T.; et al. Safety, pharmacokinetics, and pharmacodynamics of lumacaftor and ivacaftor combination therapy in children aged 2-5 years with cystic fibrosis homozygous for F508del-CFTR: An open-label phase 3 study. Lancet Respir. Med. 2019, 7, 325-335. [CrossRef]

50. Hubert, D.; Chiron, R.; Camara, B.; Grenet, D.; Prévotat, A.; Bassinet, L.; Dominique, S.; Rault, G.; Macey, J.; Honoré, I.; et al. Real-life initiation of lumacaftor/ivacaftor combination in adults with cystic fibrosis homozygous for the Phe508del CFTR mutation and severe lung disease. J. Cyst. Fibros. 2017, 16, 388-391. [CrossRef] [PubMed]

51. Cholon, D.M.; Quinney, N.L.; Fulcher, M.L.; Esther CRJr Das, J.; Dokholyan, N.V.; Randell, S.H.; Boucher, R.C.; Gentzsch, M. Potentiator ivacaftor abrogates pharmacological correction of $\triangle$ F508 CFTR in cystic fibrosis. Sci. Transl. Med. 2014, 6, 246 ra96. [CrossRef]

52. Schneider, E.K. Cytochrome P450 3A4 Induction: Lumacaftor versus Ivacaftor Potentially Resulting in Significantly Reduced Plasma Concentration of Ivacaftor. Drug Metab. Lett. 2018, 12, 71-74. [CrossRef] [PubMed]

53. European Medicines Agency. Symkevi. Available online: https://www.ema.europa.eu/en/medicines/human/EPAR/symkevi (accessed on 1 September 2020).

54. Food and Drug Administration. Symdeko. Available online: https://www.accessdata.fda.gov/drugsatfda_docs/label/2018/210 491lbl.pdf (accessed on 1 September 2020).

55. Taylor-Cousar, J.L.; Munck, A.; McKone, E.F.; van der Ent, C.K.; Moeller, A.; Simard, C.; Wang, L.T.; Ingenito, E.P.; McKee, C.; Lu, Y.; et al. Tezacaftor-Ivacaftor in Patients with Cystic Fibrosis Homozygous for Phe508del. N. Engl. J. Med. 2017, 377, 2013-2023. [CrossRef]

56. Rowe, S.M.; McColley, S.A.; Rietschel, E.; Li, X.; Bell, S.C.; Konstan, M.W.; Marigowda, G.; Waltz, D.; Boyle, M.P.; VX09-809-102 Study Group. Lumacaftor/Ivacaftor Treatment of Patients with Cystic Fibrosis Heterozygous for F508del-CFTR. Ann. Am. Thorac. Soc. 2017, 14, 213-219. [CrossRef] [PubMed] 
57. Shteinberg, M.; Taylor-Cousar, J.L. Impact of CFTR modulator use on outcomes in people with severe cystic fibrosis lung disease. Eur. Respir. Rev. 2020, 29, 190112. [CrossRef]

58. Rowe, S.M.; Daines, C.; Ringshausen, F.C.; Kerem, E.; Wilson, J.; Tullis, E.; Nair, N.; Simard, C.; Han, L.; Ingenito, E.P.; et al. Tezacaftor-Ivacaftor in Residual-Function Heterozygotes with Cystic Fibrosis. N. Engl. J. Med. 2017, 377, 2024-2035. [CrossRef]

59. Walker, S.; Flume, P.; McNamara, J.; Solomon, M.; Chilvers, M.; Chmiel, J.; Harris, R.S.; Haseltine, E.; Stiles, D.; Li, C.; et al. A phase 3 study of tezacaftor in combination with ivacaftor in children aged 6 through 11 years with cystic fibrosis. J. Cyst. Fibros. 2019, 18, 708-713. [CrossRef] [PubMed]

60. Southern, K.W.; Murphy, J.; Sinha, I.P.; Nevitt, S.J. Corrector therapies (with or without potentiators) for people with cystic fibrosis with class II CFTR gene variants (most commonly F508del). Cochrane Database Syst. Rev. 2020, 12, CD010966. [CrossRef]

61. Munck, A.; Kerem, E.; Ellemunter, H.; Campbell, D.; Wang, L.T.; Ahluwalia, N.; Owen, C.A.; Wainwright, C. Tezacaftor/ivacaftor in people with cystic fibrosis heterozygous for minimal function CFTR mutations. J. Cyst. Fibros. 2020, 19, 962-968. [CrossRef]

62. Taylor-Cousar, J.L.; Mall, M.A.; Ramsey, B.W.; McKone, E.F.; Tullis, E.; Marigowda, G.; McKee, C.M.; Waltz, D.; Moskowitz, S.M.; Savage, J.; et al. Clinical development of triple-combination CFTR modulators for cystic fibrosis patients with one or two F508delalleles. ERJ Open Res. 2019, 5, 00082-02019. [CrossRef]

63. Vertex Pharmaceuticals Incorporated. A Study Evaluating the Safety of VX-152 Combination Therapy in Adults with Cystic Fibrosis. NLM Identifier: NCT02951195. Available online: https:/ / clinicaltrials.gov/ct2/show / NCT02951195 (accessed on 30 September 2020).

64. Vertex Pharmaceuticals Incorporated. A Study Evaluating the Safety and Efficacy of VX-440 Combination Therapy in Subjects with Cystic Fibrosis. NLM Identifier: NCT02951182. Available online: https:/ / clinicaltrials.gov/ct2/show/results/NCT02951182 (accessed on 30 September 2020).

65. European Medicines Agency. Kaftrio. Available online: https://www.ema.europa.eu/en/medicines/human/EPAR/kaftrio (accessed on 28 September 2020).

66. Vertex Pharmaceuticals Inc. Trikafta ${ }^{\mathrm{TM}}$ (Elexacaftor, Tezacaftor and Ivacaftor Tablets; Ivacaftor Tablets): US Prescribing Information. 2019. Available online: https:/ / pi.vrtx.com/files/uspi_elexacaftor_tezacaftor_ivacaftor.pdf (accessed on 15 November 2020).

67. Keating, D.; Marigowda, G.; Burr, L.; Daines, C.; Mall, M.A.; McKone, E.F.; Ramsey, B.W.; Rowe, S.M.; Sass, L.A.; Tullis, E.; et al. VX-445-Tezacaftor-Ivacaftor in Patients with Cystic Fibrosis and One or Two Phe508del Alleles. N. Engl. J. Med. 2018, 379, 1612-1620. [CrossRef]

68. Heijerman, H.G.M.; McKone, E.F.; Downey, D.G.; Van Braeckel, E.; Rowe, S.M.; Tullis, E.; Mall, M.A.; Welter, J.J.; Ramsey, B.W.; McKee, C.M.; et al. Efficacy and safety of the elexacaftor plus tezacaftor plus ivacaftor combination regimen in people with cystic fibrosis homozygous for the F508del mutation: A double-blind, randomised, phase 3 trial. Lancet 2019, 394, 1940-1948, Erratum in: Lancet 2020, 395, 1694. [CrossRef]

69. Middleton, P.G.; Mall, M.A.; Dřevínek, P.; Lands, L.C.; McKone, E.F.; Polineni, D.; Ramsey, B.W.; Taylor-Cousar, J.L.; Tullis, E.; Vermeulen, F.; et al. Elexacaftor-Tezacaftor-Ivacaftor for Cystic Fibrosis with a Single Phe508del Allele. N. Engl. J. Med. 2019, 381, 1809-1819. [CrossRef] [PubMed]

70. Griese, M.; Costa, S.; Linnemann, R.W.; Mall, M.A.; McKone, E.F.; Polineni, D.; Quon, B.S.; Ringshausen, F.C.; Taylor-Cousar, J.L.; Withers, N.J.; et al. Safety and Efficacy of Elexacaftor/Tezacaftor/Ivacaftor for 24 Weeks or Longer in People with Cystic Fibrosis and One or More F508del Alleles: Interim Results of an Open-Label Phase 3 Clinical Trial. Am. J. Respir. Crit. Care Med. 2021, 203, 381-385. [CrossRef] [PubMed]

71. Burgel, P.R.; Durieu, I.; Chiron, R.; Ramel, S.; Danner-Boucher, I.; Prevotat, A.; Grenet, D.; Marguet, C.; Reynaud-Gaubert, M.; Macey, J.; et al. Rapid Improvement after Starting Elexacaftor-tezacaftor-ivacaftor in Patients with Cystic Fibrosis and Advanced Pulmonary Disease. Am. J. Respir. Crit. Care Med. 2021, 204. [CrossRef]

72. Zemanick, E.T.; Taylor-Cousar, J.L.; Davies, J.; Gibson, R.L.; Mall, M.A.; McKone, E.F.; McNally, P.; Ramsey, B.W.; Rayment, J.H.; Rowe, S.M.; et al. A Phase 3 Open-Label Study of ELX/TEZ/IVA in Children 6 through 11 Years of Age with CF and at Least One F508del Allele. Am. J. Respir. Crit. Care Med. 2021, 203. [CrossRef]

73. Van Goor, F. Precision Medicine in Cystic Fibrosis: Ivacaftor in Rare Mutations. In Proceedings of the 30th North American Cystic Fibrosis Conference. Cystic Fibrosis Foundation, Orlando, FL, USA, 27-29 October 2016.

74. Davies, J.C.; Moskowitz, S.M.; Brown, C.; Horsley, A.; Mall, M.A.; McKone, E.F.; Plant, B.J.; Prais, D.; Ramsey, B.W.; Taylor-Cousar, J.L.; et al. VX-659-Tezacaftor-Ivacaftor in Patients with Cystic Fibrosis and One or Two Phe508del Alleles. N. Engl. J. Med. 2018, 379, 1599-1611. [CrossRef] [PubMed]

75. Vertex Pharmaceuticals Incorporated. A Study of VX-659 Combination Therapy in CF Subjects Homozygous for F508del (F/F). NLM Identifier: NCT03460990. Available online: https:/ / clinicaltrials.gov/ct2/show / NCT03460990 (accessed on 1 October 2020).

76. Vertex Pharmaceuticals Incorporated. A Phase 3 Study of VX-659 Combination Therapy in Subjects with Cystic Fibrosis Heterozygous for the F508del Mutation and a Minimal Function Mutation (F/MF). NLM Identifier: NCT03447249. Available online: https: / / clinicaltrials.gov/ct2/show / NCT03447249 (accessed on 1 October 2020).

77. Giuliano, K.A.; Wachi, S.; Drew, L.; Dukovski, D.; Green, O.; Bastos, C.; Cullen, M.D.; Hauck, S.; Tait, B.D.; Munoz, B.; et al. Use of a high-throughput phenotypic screening strategy to identify amplifiers, a novel pharmacological class of small molecules that exhibit functional synergy with potentiators and correctors. SLAS Discov. 2018, 23, 111-121. [CrossRef] [PubMed] 
78. Molinski, S.V.; Ahmadi, S.; Ip, W.; Ouyang, H.; Villella, A.; Miller, J.P.; Lee, P.; Kulleperuma, K.; Du, K.; Di Paola, M.; et al. Orkambi ${ }^{\circledR}$ and amplifier co-therapy improves function from a rare CFTR mutation in gene-edited cells and patient tissue. EMBO Mol. Med. 2017, 9, 1224-1243. [CrossRef]

79. Bardin, E.; Pastor, A.; Semeraro, M.; Golec, A.; Hayes, K.; Chevalier, B.; Berhal, F.; Prestat, G.; Hinzpeter, A.; Gravier-Pelletier, C.; et al. Modulators of CFTR. Updates on clinical development and future directions. Eur. J. Med. Chem. 2021, 213, 113195. [CrossRef] 